\title{
The effect of gelatin-chondroitin sulfate-hyaluronic acid skin substitute on wound healing in SCID mice
}

\author{
Tzu-Wei Wang ${ }^{\mathrm{a}}$, Jui-Sheng Sun ${ }^{\mathrm{b}, \mathrm{c}}$, Hsi-Chin $\mathrm{Wu}^{\mathrm{a}}$, Yang-Hwei Tsuang ${ }^{\mathrm{c}}$, \\ Wen-Hsi Wang ${ }^{\text {a }}$, Feng-Huei Lin ${ }^{\mathrm{a}, *}$ \\ ${ }^{a}$ Institute of Biomedical Engineering, College of Medicine and College of Engineering, National Taiwan University, Taipei, Taiwan \\ ${ }^{\mathrm{b}}$ Institute of Rehabilitation Science and Technology, National Yang-Ming University, Taipei, Taiwan \\ ${ }^{\mathrm{c}}$ Department of Orthopedic Surgery, Taipei City Hospital, Taipei, Taiwan
}

Received 25 April 2006; accepted 17 July 2006

\begin{abstract}
Tissue-engineered skin substitutes provided a feasibility to overcome the shortage of skin autograft by culturing keratinocytes and dermal fibroblasts in vitro. In this study, we applied bi-layer gelatin-chondrointin-6-sulfate-hyaluronic acid (gelatin-C6S-HA) biomatrices onto the severe combined immunodeficiency (SCID) mice to evaluate its effect on promoting wound healing. Human foreskin keratinocytes and dermal fibroblasts were cultured with reconstructed skin equivalent (rSE) for 7 days. The rSE was then grafted to the dorsum of SCID mice to evaluate its biocompatibility by histologic and immunohistochemistry analysis. The results showed that human epidermis were well-developed with the expression of differentiated markers and basement membrane-specific proteins at 4 weeks. After implantation, the percentages of skin graft take were satisfactory, while cell-seeded group was better than noncell-seeded one. The basement membrane proteins including laminin, type IV collagen, type VII collagen, integrin $\alpha 6$, and integrin $\beta 4$ were all detected at the dermal-epidermal junction, which showed a continuous structure in the 4 weeks after grafting. This bi-layer gelatin-C6S-HA skin substitute not only has positive effect on promoting wound healing, but also has high rate of graft take. This rSE would have the potential to be applied on the extensively and deeply burned patients who suffer from severe skin defect in the near future.
\end{abstract}

(C) 2006 Elsevier Ltd. All rights reserved.

Keywords: Wound healing; Transplantation; Biomimetic material

\section{Introduction}

The difficulty in the treatment of extensively and deeply burned patients has been the limited number of donor sites available for autografting. Permanent wound closure currently requires grafting of autologous epithelium to restore the epidermal function of the skin. Split-thickness skin autograft remains the treatment of choice for permanent closure of excised burns [1]. However, it has limited availability in large burns, and it generates a donor site that is painful during healing and that may scar to become a cause of long-term morbidity [2]. In response to these limitations, alternatives to autograft have been

\footnotetext{
*Corresponding author. Tel.: + 886223912641 ; fax: + 886223940049

E-mail address: double@ha.mc.ntu.edu.tw (F.-H. Lin).
}

developed to replace either by the epidermal or dermal component of skin in separate surgical procedures [3-5]. Allografts obtained from human cadaver skin, xenografts from animal skin, or synthetic wound dressings could be used to rapidly cover burn wounds. These treatments would provide only temporary protection that would necessitate to be resurfaced with autogenous epidermis layer. In addition, the allogenic and xenogenic grafts will often be rejected by the recipient due to host immunoresponse, while the synthetic wound dressings often accomplish temporary covering to the wound site.

A new avenue for wound treatment arises from the rapidly developing technology of tissue engineering [6]. The possibility to expand epidermal cells in vitro and to produce autologous cultured epithelium represents an important breakthrough in burn therapy, because it allows 
permanent coverage of large burn wounds [7,8]. However, these cultured epithelial sheets reconstruct only the epidermis. They lack dermis to provide elasticity and mechanical stability associated with the underlying connective tissues [9]. Various approaches have been designed to produce dermal substitutes in vitro including neonatal dermal fibroblasts cultured in poly(L-glutamic acid) gels or collagen sponges, and fibroblasts seeded on synthetic or polyglycolic acid meshes [10-12]. The addition of either synthetic polymers or allogenic fibroblasts in these models often results in a risk of triggering an inflammatory reactions and transferring infectious agents [13,14]. Although, many commercialized skin graft products were available nowadays, the ideal tissue-engineered skin substitute for extensively burn wounds has not been established yet [15]. Each of these materials provides certain medical advantages to patients, but none provides the permanent repair of full-thickness skin that is required for indefinite restoration of systemic homeostasis [16-20].

In this study, we apply our previously designed bi-layer gelatin-chondrointin-6-sulfate-hyaluronic acid (gelatinC6S-HA) biomatrices [21], seeded with or without kerstinocytes and dermal fibroblasts, grafting onto the dorsum of severe combined immunodeficiency (SCID) mice to evaluate the effect on promoting wound-healing process. The results showed that in addition to reforming a permanent coverage with histologically normal and adequately differentiated epithelial tissue, the presence of a well-defined dermal-epidermal junction as well as collagen network in the dermis indicated that the bi-layer gelatinC6S-HA skin substitute has positive effect on promoting wound-healing process and could be used to help the regeneration on the full-thickness skin defect.

\section{Materials and methods}

\subsection{Preparation of bi-layer gelatin-C6S-HA scaffold}

Gelatin (Cat. No. G-2500), C6S (Cat. No. C-4384), HA (Cat. No. H5388), and 1-ethyl-3 (3-dimethyl aminopropryl) carbodiimide (Cat. No. E1769) were all purchased from Sigma Chemical Co. (St. Louis, MO, USA). At $25^{\circ} \mathrm{C}, 5 \mathrm{wt} \%$ gelatin was dissolved in distilled water. The powder of C6S and HA were added to the gelatin solution with the final concentration of $0.05 \mathrm{wt} \%$ of $\mathrm{C} 6 \mathrm{~S}$ and $0.2 \mathrm{wt} \%$ of $\mathrm{HA}$, respectively. The slurry solution was then well mixed at $30^{\circ} \mathrm{C}$ by magnetic stirrer for $1 \mathrm{~h}$. Next, $0.5 \mathrm{ml}$ of the well-mixed slurry was poured into a preformed stainless-steel mold $(3 \times 3 \mathrm{~cm}$ in diameter). For the preparation of the smaller pore size upper layer $(20-50 \mu \mathrm{m})$, the formed slurry sheet was frozen to the temperature of $-196^{\circ} \mathrm{C}$ for $30 \mathrm{~s}$. To prepare larger pore $(75-$ $150 \mu \mathrm{m}$ ) size layer, another $0.5 \mathrm{ml}$ of slurry solution was added onto the top layer of the frozen membrane and was then frozen at $-80^{\circ} \mathrm{C}$ for $3 \mathrm{~h}$ followed by lyophilizing at $-70{ }^{\circ} \mathrm{C}$ overnight. The prepared bi-layer membrane was cross-linked by $0.5 \mathrm{wt} \%$ 1-ethyl-3 (3-dimethylaminopropyl) carbodiimide solution with $0.25 \mathrm{wt} \% \mathrm{~N}$-hydroxysuccinimide (Cat. No. 56480, Sigma) addition overnight at $4{ }^{\circ} \mathrm{C}$. The cross-linked membrane was finally immersed in disodium phosphate (Cat. No. S-7909, Sigma) solution, sonicated five times in distilled water for $15 \mathrm{~min}$ to remove residual carbodiimide, and was then frozen at $-80^{\circ} \mathrm{C}$ for $3 \mathrm{~h}$ followed by lyophilizing at $-70^{\circ} \mathrm{C}$.

\subsection{Culture of keratinocytes and dermal fibroblasts}

Fresh human foreskin was obtained from human circumcision surgery with informed consent of the donors. The biopsies were washed by phosphate-buffered saline with $5 \mu \mathrm{g} / \mathrm{ml}$ Gentamycin (Cat. No. 15710, Gibco Invitrogen Co., Canada). Specimens were then cut into small fragments. The fragments were immersed in $40 \mathrm{U} / \mathrm{ml}$ thermolysin (Cat. No. T-7902, Sigma) at $4{ }^{\circ} \mathrm{C}$ overnight to separate epidermis from dermis. For isolation of keratinocytes, the epidermis was incubated in trypsinEDTA (Cat. No. 25300, Gibco, Invitrogen Co.) solution for $10 \mathrm{~min}$ at $37^{\circ} \mathrm{C}$. The isolated keratinocytes were cultivated and expanded by commercially available keratinocyte-SFM with indicated medium supplement (Cat. No. 17005, Gibco Invitrogen Co.) in a T75 culture flask. The dermis was treated with $0.2 \%$ collagenase (Cat. No. C-0130, Sigma) at $37^{\circ} \mathrm{C}$ for $2 \mathrm{~h}$ to harvest dermal fibroblasts. The harvested dermal fibroblasts were cultured in Dulbecco's Modified Eagle's Medium (DMEM) (Cat. No. SH30022.02, HyClone, Utah, USA) supplemented with 10\% FBS (Cat. No. 12003-500M, JRH Bioscience, Kansas, USA) at $37^{\circ} \mathrm{C}, 5 \% \mathrm{CO}_{2}$. Passages two to three were used for the experiments.

\subsection{Skin equivalent preparation}

In brief, the dermal fibroblasts were dynamically seeded in a selfdesigned $125 \mathrm{ml}$ spinner flask with $80 \mathrm{ml}$ medium and cell density at $2 \times 10^{5}$ cells $/ \mathrm{ml}$ for 2 days to allow for dermis development. The membrane was then taken out, and the $1 \mathrm{ml}$ of well-mixed keratinocyte cell suspension containing culture medium at a density of $2 \times 10^{6}$ cells $/ \mathrm{ml}$ was sprayed directly onto the surface of gelatin-C6S-HA membrane for epidermis development. The skin equivalent was cultured in submerge condition for 3 days in keratinocyte serum-free medium with $1 \%$ FBS addition for cell proliferation and basal lamina formation, and then transferred to air-liquid interface for further differentiation in more 2 days prior transplantation.

\subsection{Grafting of bi-layer gelatin-C6S-HA skin equivalent}

Adult CB17/ICR-SCID female mice with the age of 6 weeks old and $21 \pm 2.3 \mathrm{~g}$ in weight were used as graft recipients. They were purchased from National Laboratory Animal Center of Taiwan and were maintained under sterile housing conditions. The animal study had been approved by the Research Ethical Committee of National Taiwan University (NSC 942120-M002-015). In brief, the mice were anesthetized with IMALGENE ${ }^{\circledR}$ (Merial, Lyon, France) in a dosage of $150 \mathrm{mg} / \mathrm{kg}$ according to the instruction of intraperitoneal injection. In the experiment group, the bilayer gelatin-C6S-HA skin substitute seeded with or without cells was grafted onto the dorsal muscular bed with a wound area of $1.5 \mathrm{~cm}$ in diameter as previously described (Fig. 1A) [22]. Silicone gauze with antibiotics was applied on the outmost of skin equivalent to prevent from infections. Commercialized Framycin Gauze Dressings (Standard Chem.\&Pharm. Co., Ltd., Taiwan, ROC) were used as control group (Fig. 1B). A total of 60 mice were grafted, and mice were inspected regularly for visual sign of necrosis. Mice were sacrificed for histological analysis after grafting for 4 days, 1, 2, 3, and 4 weeks, respectively.

\subsection{Histology and immunohistochemistry analysis}

For histological analysis, biopsies were excised, fixed in 4\% paraformaldehyde, and paraffin embedded. After deparaffinization, sections at 4 $6 \mu \mathrm{m}$ thickness were used for standard hematoxylin-eosin $(\mathrm{H} / \mathrm{E})$ staining and for immunohistochemistry analysis. Basement membrane proteins of laminin, type IV collagen, type VII collagen, integrin $\alpha 6$, integrin $\beta 4$, cytoskeleton proteins of keratin, cell-cell adhesion protein of E-cadherin, and extracellular matrix (ECM) protein of type I collagen were examined. Basal keratinocyte stem cell marker p63 was also demonstrated to confirm the existence of proliferating keratinocytes. The primary antibodies used in this study were listed in Table 1 . 

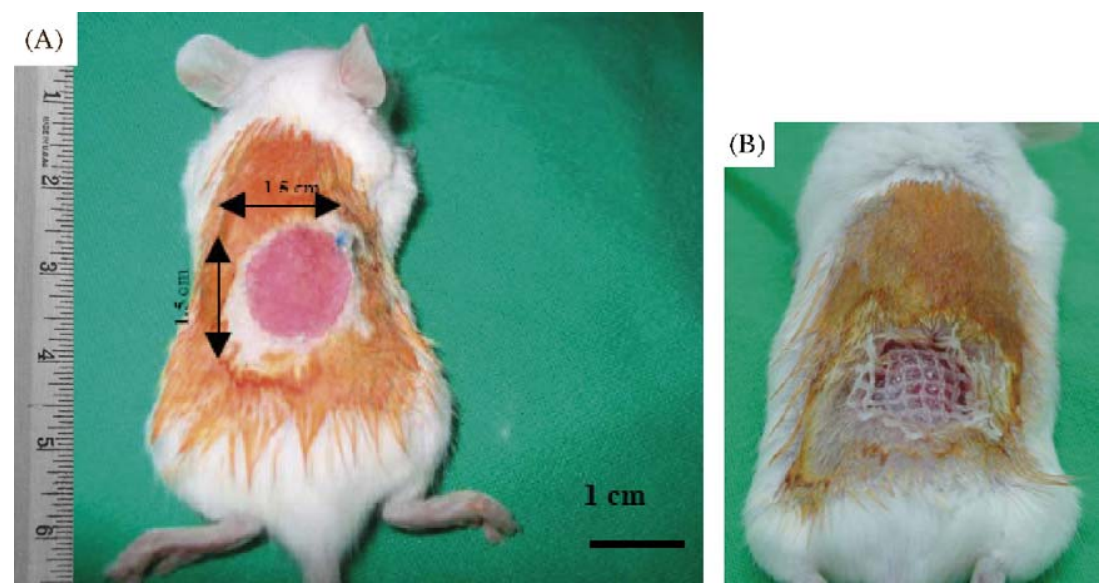

Fig. 1. The grafting process of bi-layer gelatin-C6S-HA skin substitute onto the dorsum of SCID mice. (A) A wound size of $1.5 \mathrm{~cm}$ in diameter was created on the dorsum of SCID mouse. (B) Commercialized framycin gauze dressing was used as control group.

Table 1

Lists of primary antibody and its specificity used in immunohistochemistry

\begin{tabular}{llll}
\hline Antigen & Antibody specificity & Dilution \\
\hline p63 & Monoclonal (human, rat, mouse) & $1: 1000$ & Source \\
Laminin & Monoclonal (human, pig, cat) & $1: 1000$ & Sigma, USA \\
Type IV collagen & Monoclonal (human, monkey) & $1: 500$ & Sigma, USA \\
Type VII collagen & Monoclonal (human, pig, bovine, sheep, goat) & $1: 1000$ & Sigma, USA \\
Integrin, $\alpha 6$ & Monoclonal (human) & $1: 1000$ & Sigma, USA \\
Integrin, $\beta 4$ & Monoclonal (human) & $1: 1000$ & Abcam, UK \\
Keratin & Monoclonal (human, bovine) & $1: 200$ & Abcam, UK \\
E-cadherin & Polyclonal (human) & $1: 30$ & Sigma, USA \\
Type I collagen & Monoclonal (human, rat, rabbit, pig, cow, deer) & $1: 2000$ & Abcam, UK \\
\hline
\end{tabular}

Samples were processed with heat epitope retrieval method in citrate buffer (Cat. No. 00-5000, Zymed Lab., CA, USA) to retrieve immunoreactivity. After incubation with primary antibodies, sections were stained with commercialized Super-Sensitive Non-Biotin HRP Detection System (BioGenex, CA, USA). All sections were counterstained with hematoxylin. At least three sections from three different blocks per grafted tissue were studied.

\subsection{Cytogenetic analysis}

The central region of newly synthesized skin tissue was retrieved and then the tissue was treated with $0.1 \mu \mathrm{g} / \mathrm{ml}$ microtubule stabilizer colcemid solution (Cat. No. C9754, Sigma) for $1 \mathrm{~h}$ at $37^{\circ} \mathrm{C}$ to arrest cells in metaphase. The specimen was then incubated with $2 \mathrm{wt} \%$ type I collagenase solution (Cat. No. C-0130, Sigma) for $2 \mathrm{~h}$ at $37^{\circ} \mathrm{C}$ to dissociate solid specimen by enzymatic disaggregation followed by $0.05 \%$ trypsin-EDTA solution (Cat. No. 25300, Gibco, Invitrogen Co.) for $15 \mathrm{~min}$ at $37{ }^{\circ} \mathrm{C}$ to isolate and resuspend cells. The cells were then hypotonically treated using $0.075 \mathrm{M} \mathrm{KCl}$ (Cat. No. 31248, Riedel-de Haën, Seelze, Germany) for $30 \mathrm{~min}$ at $37^{\circ} \mathrm{C}$ to enhance chromosome morphology and to increase chromatin accessibility for appropriate fixation. Sample was then immersed in methanol/acetic acid fixative in a volume ratio of 3:1 to maintain cellular architecture and to ensure that cellular proteins do not interfere with subsequent detection steps. One to two drops of the cytogenetic suspension was added onto the slide and the slide was heated to dry. About $0.4 \mathrm{wt} \%$ Geimsa solution (Cat. No. GS500, Sigma) was used for staining, and the slide was incubated for $10 \mathrm{~min}$ at room temperature followed by rinsed in water and visualized by light microscopy.

\section{Results}

\subsection{Macroscopic observation}

The results after transplantation of bi-layer gelatin-C6SHA skin substitute with different time period was demonstrated in Fig. 2. At day 0, the keratinocytes and dermal fibroblasts (K\&FB) cell-seeded group (Fig. 2A) was observed brighter and looked like more vivid in comparison with the non-cell-seeded one (Fig. 2B), in which only acellular scaffold was observed. As time went by, the graft take in cell-seeded group (Fig. 2C and E) was much higher than non-cell-seeded group (Fig. 2D and F) from 4 days to 1 week. The wound area was greatly shrunk in the cellseeded group at 2 weeks (Fig. 2G), while the graft take just started to be obviously improved in the scaffold group (Fig. 2H). At the 3 weeks, there was no wound to be seen in cell-seeded group (Fig. 2I), while there was still a small one in the scaffold group (Fig. 2J). However, there appeared to be neither scar formation nor tissue adhesion in both groups. In the 4 weeks, the skin appearance of cell-seeded group was intact with good mechanical strength (Fig. 2K), while there was an area without hairs on the dorsum of mice in the acellular scaffold group (Fig. 2L). 

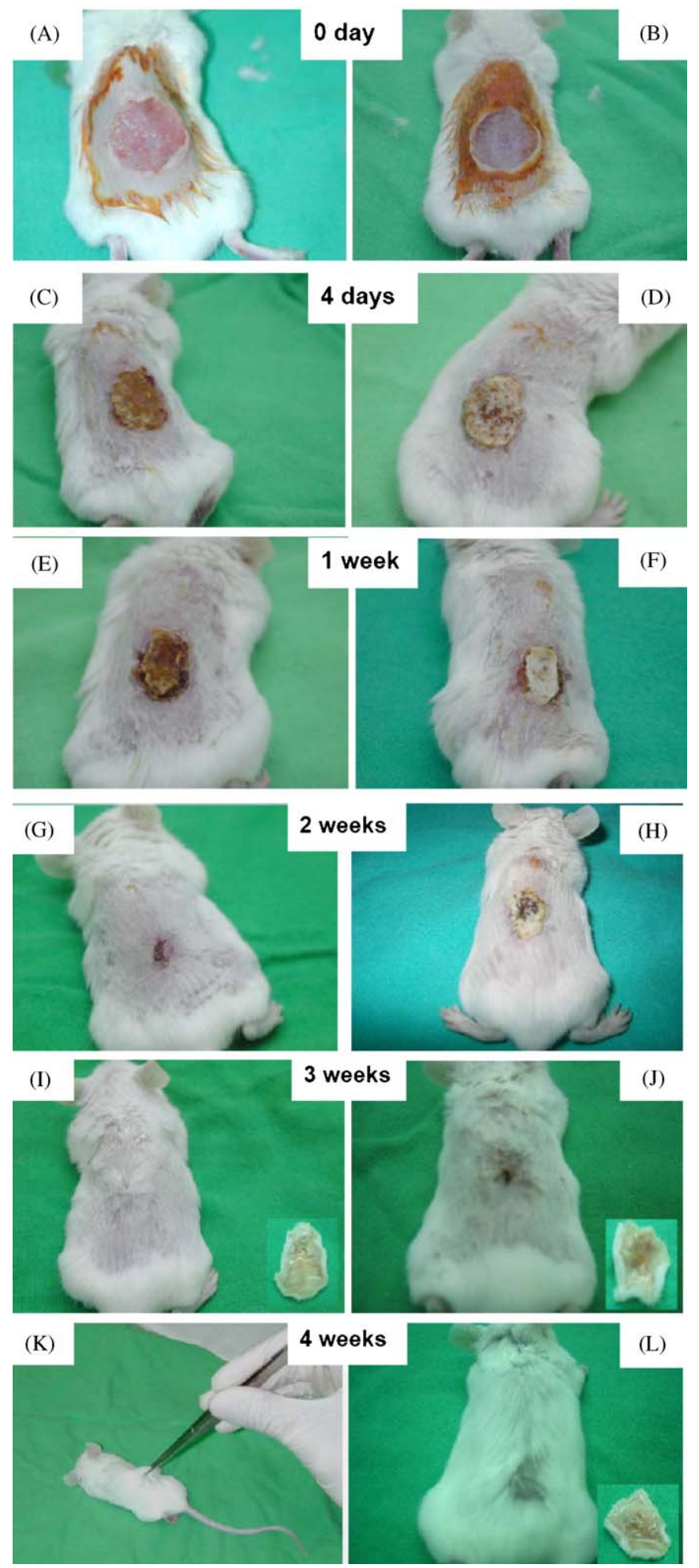

Fig. 2. The results of macroscopic observation of bi-layer gelatin-C6SHA skin substitutes in K\&FB cell-seeded group (A, C, E, G, I, K) and scaffold group (B, D, F, H, J, L) after grafting for 0 day, 4 days, 1 week, 2 weeks, 3 weeks, and 4 weeks, respectively $(N=4)$.

\subsection{Take percentages in the full-thickness skin defect after transplantation}

The graft take was assessed and defined as the intimate bio-integration of bi-layer gelatin-C6S-HA skin substitute with the wound bed, as confirmed by bright-field microscope and computer algorithm (Optimas 5.2 software, Media Cybernetics, Silver Spring, MD). The take percentages in both groups were all increased with the time as shown in Table 2. The take percentage of cell-seeded group was much higher compared to that of scaffold group from the beginning of 4 days to the end of 4 weeks. The value is from $14 \%$ to $98 \%$ in cell-seeded group, while it is from $6 \%$ to $90 \%$ in scaffold group. The take rate in cell-seeded group was really satisfactory nearly total integrated in the 4 weeks.

\subsection{Wound-healing process}

The skin equivalent grafted mice were sacrificed to evaluate the wound-healing process in the 2 weeks. Both the epithelial tissue and dermal tissue were regenerated from the underneath wound bed, and cell migration from the both sides of wound margin to the central of wound area was observed (Fig. 3A). The wound margin was amplified in the Fig. 3B. Epithelial tongue was noticed at the edge of the wound margin, which meant the keratinocytes located at the wound edge migrated and tried to cover the wound area and close the open wound during the wound-healing process.

\subsection{Identification of the origin of newly synthesized skin tissue}

Monoclonal primary antibodies of human keratin and human type I collagen were co-localized to identify whether the newly synthesized skin tissue was derived from inoculated human cells.

In the Fig. 4, the wound was already re-epithelialized in the 3 weeks after transplantation though the underlying dermis tissue was not fully regenerated. The newly synthesized skin tissue including epidermis and dermis were stained in dark brown color, which suggested that the regenerated skin tissue was human origin but not from mouse. In the cytogenetic analysis, the chromosomes of cells in newly synthesized skin tissue were also extracted and the number of chromosome was counted with Giemsa stain to further confirm the cell source. The results showed that there were exactly 46 chromosomes as that of normal human beings (Fig. 5).

\subsection{Histology examination of skin morphology}

In the $\mathrm{H} / \mathrm{E}$ stain, some granulation tissue appeared in the dermal layer of the scaffold group (Fig. 6A), only mild inflammation reaction was observed in the first 2 weeks. In 3 weeks, the skin morphology was quite normal with adequate thickness of epidermal layer and dermal layer (Fig. 6B). The granulation tissue was not observed in the cell-seeded group with numerous vascular-like structures were formed in the dermal layer within 2 weeks (Fig. 6C). The epidermis was epithelialized, the dermis was 
Table 2

Take percentages in the full-thickness skin defect after transplantation

\begin{tabular}{|c|c|c|c|c|c|}
\hline \multirow[t]{2}{*}{ Groups } & \multicolumn{5}{|c|}{ Time $($ mean \pm SD $(\%))$} \\
\hline & 4 days & 1 week & 2 weeks & 3 weeks & 4 weeks \\
\hline Scaffold & $6 \pm 3$ & $25 \pm 6$ & $41 \pm 7$ & $72 \pm 5$ & $90 \pm 6$ \\
\hline K\&FB cells + scaffold & $14 \pm 5$ & $33 \pm 4$ & $79 \pm 9$ & $95 \pm 4$ & $98 \pm 2$ \\
\hline
\end{tabular}

One-Way ANOVA, SD: standard deviation, $N=4,{ }^{*} p<0.05$, all are significantly different.
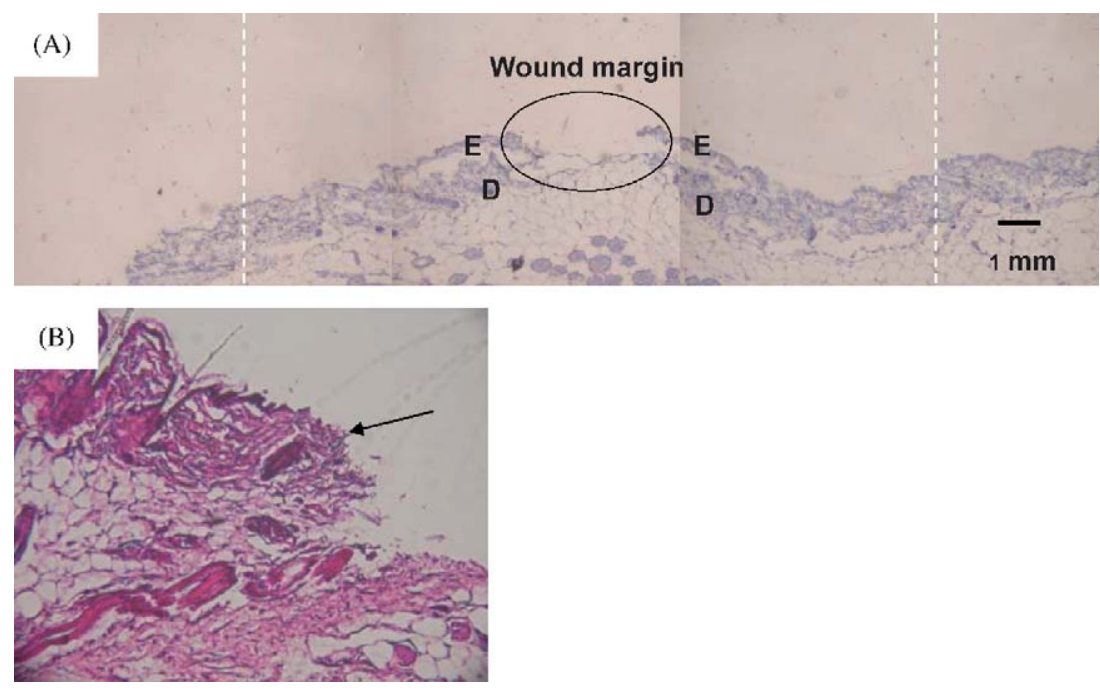

Fig. 3. (A) The wound healing process of cell-seeded rSE grafted onto SCID mice in 2 weeks. The white dash line indicated the exact wound area created on the dorsum of SCID mice. (B) The epithelial tongue (arrow indicated) was seen at the wound margin, which represented the migration of keratinocytes from surrounding tissue to wound bed $(\times 40, \mathrm{H} / \mathrm{E}$ stain) (E: epidermis, D: dermis).

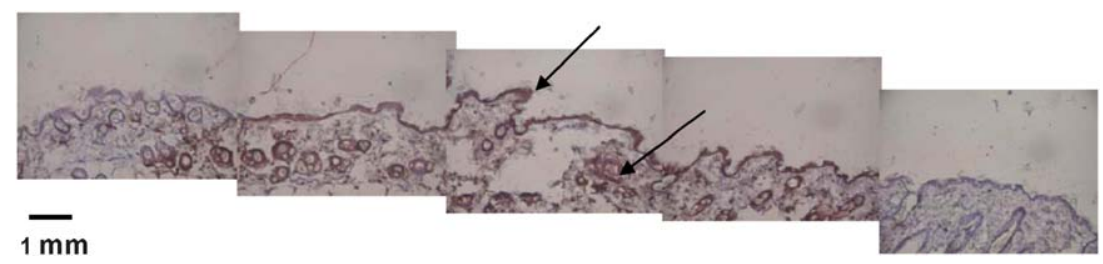

Fig. 4. The specific immunostain of human keratin in epidermis and type I collagen in dermis (arrow indicated: brown color) was used to prove the cell source of re-constructed skin tissue was derived from cultured human cells not from mouse origin after grafting for 3 weeks. The newly synthesized skin tissue in the wound area was positively stained with brown color, while the mouse skin tissue at both ends was negatively stained in light-blue hematoxylin color.

regenerated, and the hair follicles were also present in the third week (Fig. 6D). In the fourth week, the skin morphology and architecture were fully developed with well-differentiated epidermal layers (Fig. 6E and F).

\subsection{Immunohistochemistry analysis}

The histology examined in the fourth week revealed numerous features of epidermal differentiation, i.e., well organized basal layer of distinct cylindrical cells, granular, and a horny layer (Fig. 7A and C). The human-specific keratin, and E-cadherin, were well-expressed in the epidermal layer (Fig. 7B and D). The type I collagen was present throughout the ECM of the dermis (Fig. 7F and H). The phenotype of keratinocytes and dermal fibroblasts were well kept as the keratin and type I collagen was distributed in epidermal layer and dermal layer.

\subsection{Basement membrane zone reconstruction}

P63 was only present in the basal layer of keratinocytes (Fig. 8A), while the laminin 5 was noted at the dermalepidermal junctions (Fig. 8B). The intracellular presence of type IV collagen and type VII collagen in basal keratinocytes was also demonstrated by specific monoclonal antibodies, respectively (Fig. 8C and D). Integrin $\alpha 6$ and integrin $\beta 4$ exclusively located in the mature epithelium and contributing to the anchorage of basal keratinocytes to the 


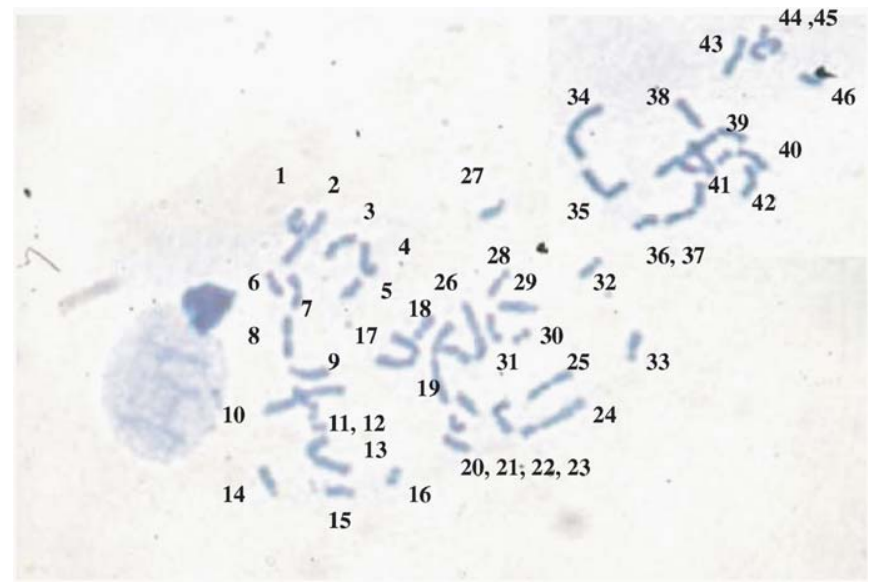

Fig. 5. The result of cytogenetic analysis. The chromosomes of cells in newly synthesized skin tissue were extracted and the number of chromosome was counted with Giemsa stain to further confirm the cell source. The number of chromosomes was exactly 46. underlying basement membrane had also been seen at the basal layer of epidermis (Fig. 8E and F).

\section{Discussion}

An ideal skin substitute should have several key attributes [23]. The dressing should protect the wound from bacterial infection, control evaporative water loss to prevent dehydration, permit oxygen and carbon dioxide exchange, absorb wound exudates, and allow the growth of skin appendages. Additionally, it should be composed of materials that are non-toxic and non-immunogenic.

In this study, C6S and HA were incorporated within the gelatin membrane to mimic skin ECM composition and to create an appropriate microenvironment for cell growth. Glycosaminoglycans not only provided the mechanical strength of the gelatin scaffold but also retained large porous lamellar matrix spaces probably due to their
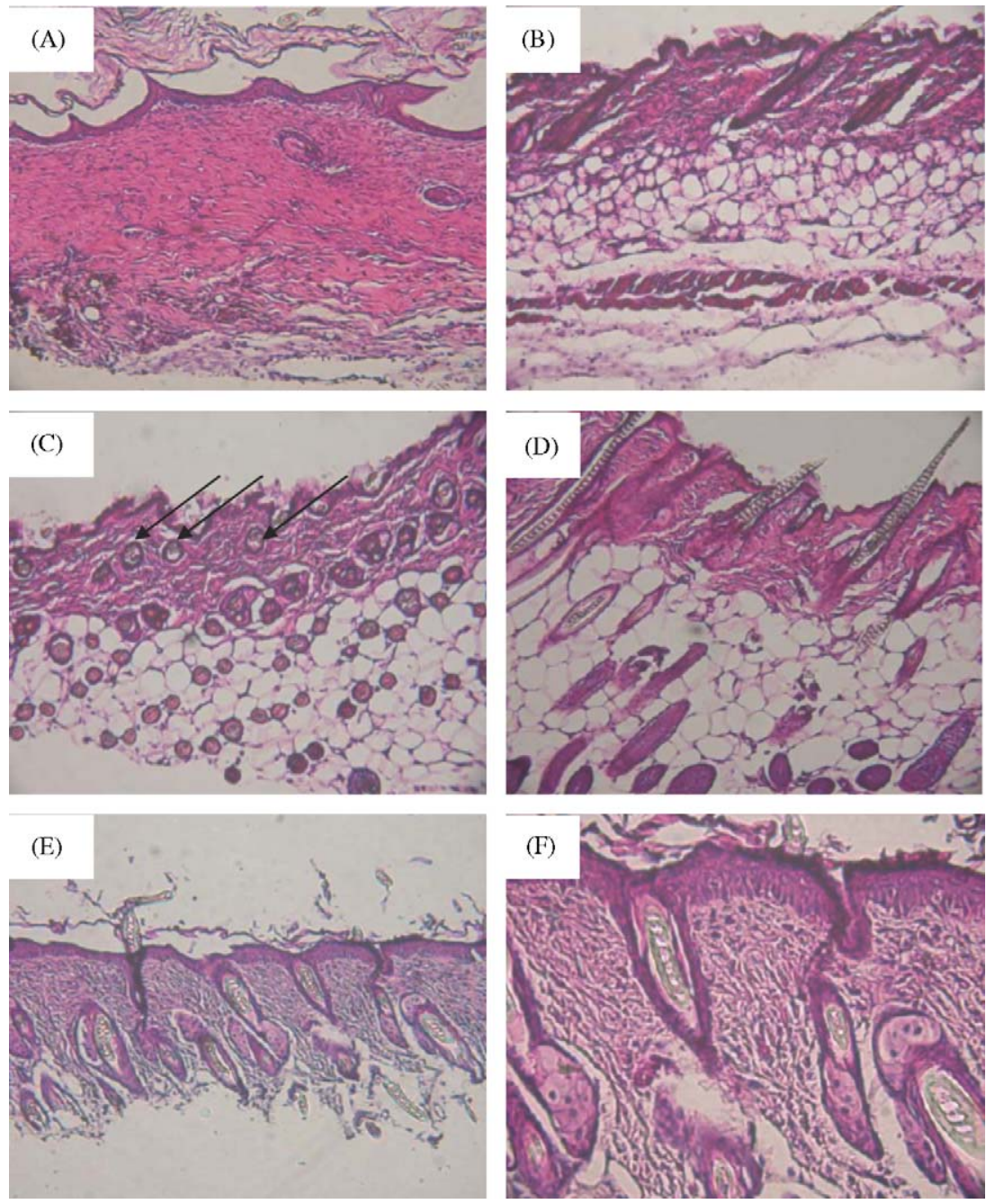

Fig. 6. The histology of grafted bi-layer skin substitute after transplantation. The scaffold group ((A) 2 weeks, (B) 3 weeks) and K\&FB cell-seeded group ((C) 2 weeks, (D) 3 weeks, (E), (F) 4 weeks). (A) Granulation tissue was formed in the scaffold group in the 2 weeks, while this phenomenon was subsided in the 3 weeks (B). (C) Vascular-like structures (arrow indicated) were appeared in the dermis and no granulation tissue has been seen in cell-seeded group in the 2 weeks. (D) The epithelium was fully re-epithelialized in the 3 weeks and the skin morphology was intact in the 4 weeks (E). (F) was the high-power view of $(\mathrm{E})(\mathrm{H} / \mathrm{E}$ stain, $(\mathrm{A})-(\mathrm{E}) \times 40, \mathrm{~F} \times 100)$. 

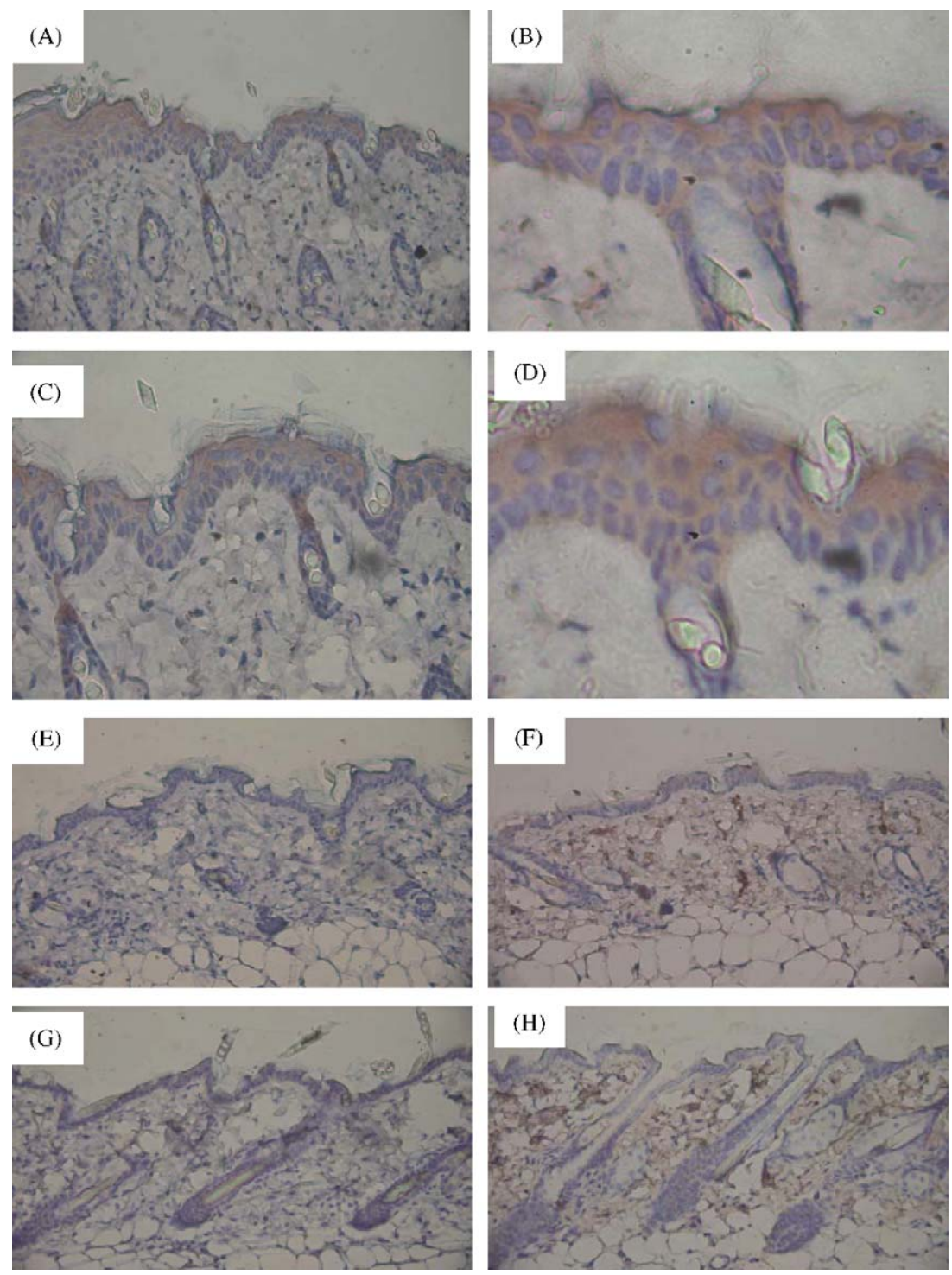

Fig. 7. Specific immunohistochemistry of human keratin ((A) 4 weeks, $\times 100$; (B) 4 weeks, $\times 400)$, E-cadherin $((C) 4$ weeks, $\times 100 ;$ (D) 4 weeks, $\times 400)$, and type I collagen $((\mathrm{F}) 2$ weeks, $\times 100 ;(\mathrm{H}) 4$ weeks, $\times 100)$. (E) and $(\mathrm{G})$ with antimouse IgG HRP secondary antibody applied, but without monoclonal primary antibody conjugated as negative control $(\times 100)$.

water-binding capacity, which would promote matrix swelling and absorb wound exudates [24]. Furthermore, the C6S and HA retained gelatin scaffold was readily biointegrated to become part of the ECM of the regenerative skin tissue (Fig. 2). The bi-layer skin equivalent was originally designed and fabricated with two different pore sizes. The pore size in the lower layer about $75-150 \mu \mathrm{m}$ frozen at $-80^{\circ} \mathrm{C}$ is considered to be suitable for dermal fibroblasts proliferation and migration. The pore size in the upper layer about $20-50 \mu \mathrm{m}$ frozen at $-196^{\circ} \mathrm{C}$ was designed for keratinocytes attachment and served to prevent keratinocytes from falling into the lower layer. The interconnected pores between two layers could provide the interaction opportunities for released cytokines and growth factors by dermal fibroblasts and epidermal keratinocytes. The pore structure of bi-layer gelatin-C6SHA membrane could be preserved in an intact form following the freeze-drying procedure that we have described in the previous study [25].

Both graft take rate and re-epithelialization have been used as measurements for monitoring wound closure and wound-healing process $[26,27]$. In this study, graft take was monitored by measuring the integration of bi-layer skin equivalent with the wound bed, and re-epithelialization was determined by measuring the advancing epithelium. Cellseeded skin equivalent had improved wound-healing process more obviously compared to wounds with scaffold alone (Table 2). In addition, the underlying vascular-like structures appeared more rapidly in cell-seeded group than in scaffold group (Fig. 6C). Re-epithelialization was the process by which new cutaneous tissue covered the wound defect [28]. This process required the uninjured keratinocytes along the wound edges to migrate laterally to cover the wound bed (Fig. 3A and B). 

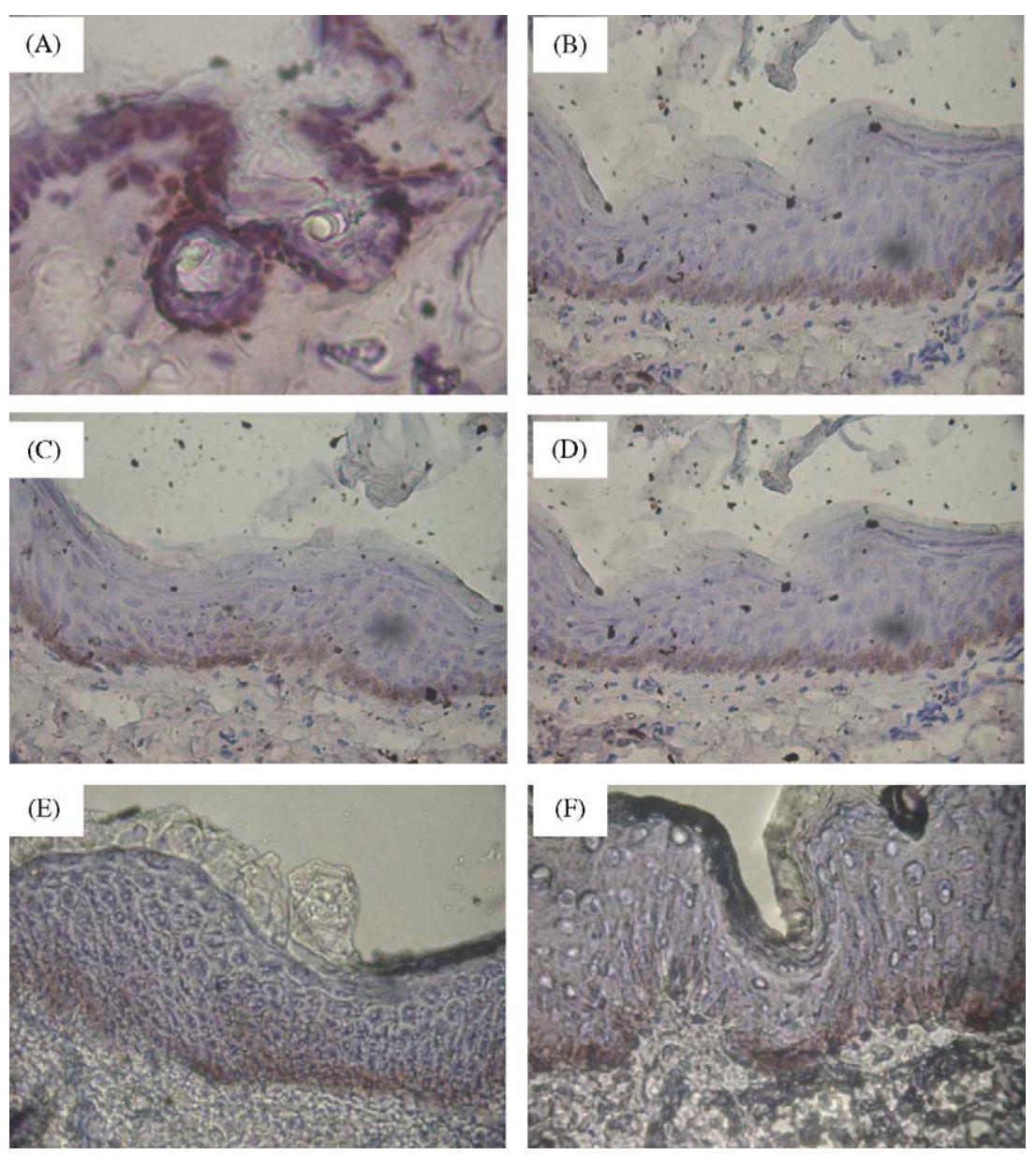

Fig. 8. The morphology and immunohistochemistry of cell-seeded skin equivalent after grafting for 4 weeks. (A) p63 was positive stained, which meant keratinocyte stem cells were located in the basal lamina. (B) Laminin was noted at the basal site of keratinocytes and represent early marker of cell polarity. (C) and (D) The intracellular presence of type IV collagen and type VII collagen in basal keratinocytes was stained in brown color. (E) Intrgrin $\alpha 6$ and $(\mathrm{F})$ integrin $\beta 4$ were also observed underlying the basement membrane $(\times 200)$.

The SCID mice were chosen as an experimental model in vivo evaluation of reconstructed skin equivalent (rSE), because they did not reject xenogeneic grafts. The detection of human-specific keratin and type I collagen on the grafts indicated that the human cells were not replaced by mouse cells (Fig. 4). The excellent take of the grafted rSE without necrosis was confirmed by macroscopic examination and image software. These observations were in accordance with the microscopic histological analysis (Fig. 6). A differentiated epidermis with cuboidal basal cells and several suprabasal layers was observed 4 weeks after grafting (Fig. 7A-D). At this time point, the epidermis reconstructed with human keratinocytes was thicker than the epidermis reconstructed without cell-seeded scaffold group. This thickness of newly synthesized epidermis about $100 \mu \mathrm{m}$ was comparable to that of normal human skin. On the other hand, the excellent quality of the adhesion between the epidermis and the dermis was probably due to the presence of laminin, types IV collagen, type VII collagens, integrin $\alpha 6$, and integrin $\beta 4$, those which organized in a continuous basement membrane zone as demonstrated by immunohistochemistry (Fig. 8). Epithelia, basement membrane, and stroma, these three critical components were combined together to contribute to scarless skin regeneration possible in this study [29].

In brief, the wound area has already been epithelialized by gross observation after grafting for 4 weeks. The take percentage of cell-seeded scaffold was obviously increased compared to the unseeded one. Furthermore, the basement membrane was formed and has been reconstructed in the dermal-epidermal junction. Basement membrane proteins including laminin, type IV collagen, type VII collagen, and $\alpha 6 \beta 4$ integrins were all positively stained in histological findings. Keratin and type I collagen were distributed in epidermal layer and dermal layer, respectively, which suggests that newly synthesized epidermal layer and dermal layer were mature and differentiated skin tissue. The keratinocytes developed into an epidermis-like structure with the formation of basal layers, suprabasal layers, and the cornified squamous layer. Dermal fibroblasts with sparsely and evenly distribution were surrounded with their own ECM. These findings suggest that the bi-layer gelatinC6S-HA skin equivalent were suitable to be used to help wound healing on the full-thickness skin defect. 


\section{Conclusion}

This newly designed tissue-engineered skin substitute not only has positive effect on promoting wound-healing process, but also has high percentage of graft take rate. The cell-seeded group was also much better than non-cellseeded group. In addition, the structure of the basement membrane zone was well-established, and the morphology of skin architecture including epidermal layer and dermal layer was also successfully formed. This gelatin-chondroitin-6-sulfate-hyaluronic acid skin equivalent would have the potential to be applied on the clinics to overcome the shortage of retrieving enough skin area for split-thickness autografting, and to heal the full-thickness skin defect of wounds in the near future.

\section{References}

[1] Loss M, Wedler V, Kunzi W, Meuli-Simmen C, Meyer VE. Artificial skin, split-thickness autograft and cultured autologous keratinocytes combined to treat a severe burn injury of $93 \%$ of TBSA. Burns 2000;26(7):644-52.

[2] Robson MC, Barnett RA, Leitch IO, Hayward PG. Prevention and treatment of postburn scars and contracture. World J Surg 1992;16(1):87-96.

[3] Heck EL, Bergstresser PR, Baxtor CR. Composite skin graft: frozen dermal allografts support the engraftment and expansion of autologous epidermis. J Trauma 1985;25(2):106-12.

[4] Burke JF, Yannas IV, Quinby Jr WC, Bondoc CC, Jung WK. Successful use of a physiologically acceptable artificial skin in the treatment of extensive burn injury. Ann Surg 1981;194(4):413-28.

[5] Bell E, Sher S, Hull B, Merrill C, Rosen S, Chamson A, et al. The reconstitution of living skin. J Invest Dermatol 1983;81(1 Suppl): 2S-10S.

[6] Black AF, Berthod F, L'Heureux N, Germain L, Auger FA. In vitro reconstruction of a human capillary-like network in a tissueengineered skin equivalent. FASEB J 1998;12(13):1331-40.

[7] Rheinwald JG, Green H. Serial cultivation of strains of human epidermal keratinocytes: the formation of keratinizing colonies from single cells. Cell 1975;6(3):331-44.

[8] Green H, Kehinde O, Thomas J. Growth of cultured human epidermal cells into multiple epithelia suitable for grafting. Proc Natl Acad Sci USA 1979;76(11):5665-8.

[9] Woodley DT, Peterson HD, Herzog SR, Stricklin GP, Burgeson RE, Briggaman RA, et al. Burn wounds resurfaced by cultured epidermal autografts show abnormal reconstitution of anchoring fibrils. JAMA 1988;259(17):2566-71.

[10] Bell E, Ehrlich HP, Buttle DJ, Nakatsuji T. Living tissue formed in vitro and accepted as skin-equivalent tissue of full thickness. Science 1981;211(4486): 1052-4.

[11] Naughton G, Mansbridge J, Gentzkow G. A metabolically active human dermal replacement for the treatment of diabetic foot ulcers. Artif Organs 1997;21(11):1203-10.

[12] Orgill DP, Butler C, Regan JF, Barlow MS, Yannas IV, Compton CC. Vascularized collagen-glycosaminoglycan matrix provides a dermal substrate and improves take of cultured epithelial autografts. Plast Reconstr Surg 1998;102(2):423-9.

[13] Pandit A, Ashar R, Feldman D, Thompson A. Investigation of acidic fibroblast growth factor delivered through a collagen scaffold for the treatment of full-thickness skin defects in a rabbit model. Plast Reconstr Surg 1998;101(3):766-75.

[14] Srivastava A, DeSagun EZ, Jennings LJ, Sethi S, Phuangsab A, Hanumadass $\mathrm{M}$, et al. Use of porcine acellular dermal matrix as a dermal substitute in rats. Ann Surg 2001;233(3):400-8.

[15] Bello YM, Falabella AF, Eaglstein WH. Tissue-engineered skin: current status in wound healing. Am J Clin Dermatol 2001;2(5): $305-13$.

[16] Gentzkow GD, Iwasaki SD, Hershon KS, Mengel M, Prendergast JJ, Ricotta JJ, et al. Use of dermagraft, a cultured human dermis, to treat diabetic foot ulcers. Diabetes Care 1996;19(4):350-4.

[17] Noordenbos J, Dore C, Hansbrough JF. Safety and efficacy of TransCyte for the treatment of partial-thickness burns. J Burn Care Rehabil 1999;20(4):275-81.

[18] Wainwright DJ. Use of an acellular allograft dermal matrix (AlloDerm) in the management of full-thickness burns. Burns 1995; 21(4):243-8.

[19] Kremer M, Lang E, Berger AC. Evaluation of dermal-epidermal skin equivalents ('composite-skin') of human keratinocytes in a collagenglycosaminoglycan matrix (Integra artificial skin). Br J Plast Surg 2000;53(6):459-65.

[20] Guerret S, Govignon E, Hartmann DJ, Ronfard V. Long-term remodeling of a bilayered living human skin equivalent (Apligraf) grafted onto nude mice: immunolocalization of human cells and characterization of extracellular matrix. Wound Repair Regen 2003;11(1):35-45.

[21] Wang TW, Wu HC, Huang YC, Sun JS, Lin FH. Biomimetic bilayered gelatin-chondroitin-6-sulfate-hyaluronic acid biopolymer as the scaffold for skin equivalent tissue engineering. Artif Organs 2006;30(3):141-9.

[22] Germain L, Guignard R, Rouabhia M, Auger FA. Early basement membrane formation following the grafting of cultured epidermal sheets detached with thermolysin or dispase. Burns 1995;21(3): $175-80$.

[23] Horch RE, Kopp J, Kneser U, Beier J, Bach AD. Tissue engineering of cultured skin substitutes. J Cell Mol Med 2005;9(3):592-608.

[24] Choi YS, Hong SR, Lee YM, Song KW, Park MH, Nam YS. Studies on gelatin-containing artificial skin, II: preparation and characterization of cross-linked gelatin-hyaluronate sponge. J Biomed Mater Res 1999;48(5):631-9.

[25] Huang YC, Wang TW, Sun JS, Lin FH. Epidermal morphogenesis in an in-vitro model using a fibroblasts-embedded collagen scaffold. J Biomed Sci 2005;12(6):855-67.

[26] Grant I, Green C, Martin R. Strategies to improve the take of commercially available collagen/glycosaminoglycan wound repair material investigated in an animal model. Burns 2001;27(7):699-707.

[27] Pouliot R, Larouche D, Auger FA, Juhasz J, Xu W, Li H, et al. Reconstructed human skin produced in vitro and grafted on athymic mice. Transplantation 2002;73(11):1751-7.

[28] Usui ML, Underwood RA, Mansbridge JN, Muffley LA, Carter WG, Olerud JE. Morphological evidence for the role of suprabasal keratinocytes in wound reepithelialization. Wound Repair Regen 2005;13(5):468-79.

[29] Yannas IV. Tissue and organ regeneration in adults. New York: Springer; 2001. 